\title{
Sind Opioide wirksam?
}

\section{Hier steht eine Anzeige.}

Fragestellung: Sind Opioide bei der Behandlung schwerwiegender Schmerzen bei Patienten mit Parkinson-Syndrom wirksam?

Hintergrund: Patienten mit Parkinson-Erkrankung leiden oft unter muskuloskelettalen Schmerzen, aber auch Schmerzen im Bereich des Abdomens und des Gesichtes. Ein Teil dieser Schmerzen wird durch die Grunderkrankung akzentuiert. Eine gute randomisierte Studie zur Therapie schwerwiegender Schmerzen bei Patienten mit Parkinson-Syndrom lag bisher nicht vor.

Patienten und Methodik: Die Phase-II-Studie schloss Parkinson-Patienten mit einem Hoehn und Yahr Stadium II bis IV ein, die auf einer Schmerzskala von 0 bis $10 \mathrm{im}$ Mittel einen Wert von 6 innerhalb von 24 Stunden aufwiesen. Die Patienten wurden über 16 Wochen entweder mit Oxycodon plus Naloxon oder Placebo behandelt. Der primäre Endpunkt war der mittlere 24 Stunden-Schmerz-Score nach 16 Wochen.

Ergebnisse: In die Studie wurden 202 im Mittel 67 Jahre alte Patienten eingeschlossen, von denen 93 das Opioid und 109 Placebo erhielten. Das Parkinson-Syndrom bestand im Mittel seit sieben Jahren, die Schmerzen seit dreieinhalb Jahren. Der mittlere 24-Stunden-Schmerz-Score betrug zu Studienbeginn 7,3. Die meisten Patienten hatten muskuloskelettale Schmerzen. Der mittlere 24-Stunden-Schmerz-Score nach 16 Wochen betrug in der Opioidgruppe 5,0, in der Placebogruppe 5,6 (nicht signifikant, $\mathrm{p}=0,058)$. Übelkeit

Trenkwalder C, Chaudhuri KR, Martinez-Martin P et al; PANDA study group. Prolonged-release oxycodone-naloxone for treatment of severe pain in patients with Parkinson's disease (PANDA): a double-blind, randomised, placebo-controlled trial. Lancet Neurol 2015; 14: 1161 - 70 und Obstipationen waren in der Verumgruppe häufiger.

Schlussfolgerungen: Diese randomisierte Studie zeigt einen Trend für den Einsatz eines Opioids bei Patienten mit Parkinson-Syndrom und ausgeprägten Schmerzen.

\section{- Kommentar von Hans-Christoph Diener, Essen}

\section{Ein positiver Trend}

Diese relativ kleine Studie zeigt einen Trend, aber keine signifikante Überlegenheit für den Einsatz eines potenten Opioids zur Behandlung ausgeprägter Schmerzen bei Patienten mit Parkinson-Syndrom. Die Tatsache, dass diese Studie negativ ausging, mag auf die relativ geringe Anzahl der Studienteilnehmer zurückzuführen sein. Weitaus bedeutsamer ist allerdings die Tatsache, dass ganz unterschiedliche Schmerzerkrankungen und Schmerztypen in diese Studie aufgenommen wurden.

Erwähnenswert ist, dass die Parkinson-Symptomatik durch den Einsatz des Opioids nicht verschlechtert wurde. 